\title{
Capacidade produtiva e progresso genético de pinhão-manso
}

\author{
Productive capacity and genetic progress of physic nut
}

\section{Catiele Vieira Borges ${ }^{\mathrm{I}}$ Fábio Medeiros Ferreira ${ }^{\mathrm{II}}$ Rodrigo Barros Rocha ${ }^{\mathrm{III}}$ Adriano Ramos dos Santos ${ }^{\mathrm{IV}}$ Bruno Galvêas Laviolav}

\section{RESUMO}

O objetivo do presente estudo foi estimar parâmetros genéticos e avaliar o progresso genético dos principais componentes da produção de grãos do pinhão-manso. Para isso, foram avaliadas quatro procedências de pinhão-manso em ensaio de blocos ao acaso, utilizando três repetições de 36 plantas, em espaçamento de $3 \times 2 \mathrm{~m}$. O número de cachos por planta foi uma das características mais importantes para a produção de grãos. A uniformidade de maturação dos frutos apresentou efeito ambiental predominante, sendo bastante limitado o progresso genético a ser obtido com a seleção baseada nesta característica. O progresso genético estimado com a seleção de procedências pode ser considerado pequeno: $11 \%, 7 \%$ e $18 \%$ no $1^{\circ}$, 2o e 3o anos pósplantio, respectivamente. A seleção dos 30 melhores indivíduos dentro de procedencias permitiu a obtenção de ganhos de $29 \%$. O melhoramento dessa oleaginosa deve considerar estratégias de geração de variabilidade, como a recombinação de indivíduos divergentes e introdução de novos acessos.

Palavras-chave: Jatropha curcas L., melhoramento genético, parâmetros genéticos.

\section{ABSTRACT}

The objective of this study was to estimate genetic parameters and evaluate the genetic progress of Jatropha curcas L. agricultural traits from 2008 to 2011. Four Brazilian provenances were evaluated in a randomized block design using three replicates of 36 plants at a spacing of $3 \times 2 \mathrm{~m}$. The number of bunches was one of the most important traits for grain production. The fruit ripening uniformity showed predominant environmental effect, being very limited the genetic progress to be achieved by plant selection. The estimated genetic progress with the selection of provenances may be considered low: $11 \%, 7 \%$ and $18 \%$ at $1^{\text {th }}, 2^{\text {th }}$ and $3^{\text {th }}$ years respectively. The selection of the 30 best individuals within provenances provided a genetic progress of 29\%. The genetic improvement of this oil crop should consider strategies to increase the genetic variability by breeding and introduction of new genotypes.

Key words: Jatropha curcas L., breeding, genetic parameters.

\section{INTRODUÇÃO}

O pinhão-manso (Jatropha curcas L., Euphorbiaceae) é originário da América Central com ocorrência em todas as regiões intertropicais e até mesmo em zonas temperadas (BHERING et al., 2013). Embora considerada uma planta com potencial para fornecer matéria-prima para fins combustíveis, essa oleaginosa ainda se encontra em fase de domesticação e busca por domínio tecnológico (LAVIOLA et al., 2011).

Segundo SPINELLI et al. (2010), a produtividade de grãos é uma das características mais importantes para seleção, estando associada a mais de $90 \%$ da variabilidade do rendimento de óleo. Produções inferiores a $3 \mathrm{t} \mathrm{ha}^{-1}$ estão sendo observadas em diferentes condições edafoclimáticas, devido a limitações hídricas, ataques de pragas e doenças e plantio de material não selecionado. A ocorrência de acessos aparentados provenientes de diferentes regiões do Brasil indica a baixa diversidade genética dessa oleaginosa no país (ROSADO et al., 2010). Para

'Faculdade de Ciências Agrárias, Universidade Federal do Amazonas (UFAM), 69077-000, Manaus, AM, Brasil. E-mail: catiele.borges@ufam.edu.br. Autor para correspondência.

"Instituto de Ciências Exatas e Tecnologia (ICET), UFAM, Itacoatiara, AM, Brasil.

IIIEmpresa Brasileira de Pesquisa Agropecuária (EMBRAPA), Porto Velho, RO, Brasil.

${ }^{\text {IV }}$ Centro de Estudos da Cultura e do Meio Ambiente da Amazônia (RIOTERRA), Porto Velho, RO, Brasil.

${ }^{v}$ Embrapa Agroenergia, Brasília, DF, Brasil. 
BASHA et al. (2009), a baixa diversidade genética é consequência do número reduzido de introduções de J. curcas no país.

Por se tratar de um cultivo perene em fase de domesticação, a avaliação de plantas em idade produtiva ao longo do tempo é importante para o melhoramento do pinhão-manso (RESENDE, 2007). Recursos genéticos utilizados para seleção de plantas podem ser organizados em grupos que compreendem de espécies silvestres a variedades modernas. Testes de procedências, em que cada população é representada por uma mistura de sementes de várias matrizes, são recursos genéticos importantes na seleção dessa oleaginosa em domesticação que não possui variedades comerciais (LAVIOLA et al., 2011; ROCHA et al., 2009).

A seleção de plantas baseia-se nos valores genéticos aditivos das matrizes que serão recombinadas e nos valores genotípicos das plantas que podem ser propagadas vegetativamente. As estimativas dos parâmetros genéticos são importantes para: a caracterização da estrutura genética da população, a inferência da variabilidade genética e a predição dos ganhos de seleção (CRUZ \& CARNEIRO, 2003). Dentre os principais procedimentos para a estimação dos parâmetros genéticos, destacam-se a metodologia de modelos mistos para obtenção do melhor preditor linear não viesado (BLUP) e o procedimento de máxima verossimilhança restrita (REML) para obtenção dos componentes de variância.

Esse procedimento tem se consolidado na avaliação genética de espécies perenes, por permitir a predição de valores genéticos associado às observações fenotípicas, ajustando-se os dados aos efeitos fixos e ao número desigual de informações por parcela (RESENDE, 2007). Na seleção de plantas de maior estabilidade e adaptabilidade, tem sido utilizado o método da média harmônica da performance relativa dos valores genéticos preditos (MHPRVG). Esse método, proposto por RESENDE (2007), permite realizar a seleção simultânea de plantas de desempenho superior e de estabilidade produtiva, similarmente à metodologia de LIN \& BINNS (1988), agregando as vantagens dos modelos mistos.

O objetivo deste trabalho foi selecionar as melhores procedências de pinhão-manso, considerando seus parâmetros genéticos, e avaliar o progresso genético dos principais componentes da produção de grãos no $1^{\circ}, 2^{\circ}$ e $3^{\circ}$ ano pós-plantio.

\section{MATERIAL E MÉTODOS}

No mês de março de 2008, foi instalado no campo experimental do Centro de Pesquisas
Agroflorestais da Empresa Brasileira de Pesquisa Agropecuária (EMBRAPA-CPAFRO), Porto Velho$\mathrm{RO}$, um teste de procedências de pinhão-manso pertencente aos estados de Rondônia (1 e 2), Minas Gerais (3) e São Paulo (4), com 108 plantas por procedência, obtidas a partir de sementes de origem genética desconhecida. Sementes dessas populações estão representadas no Banco de Germoplasma de pinhão-manso, instalado na Embrapa Agroenergia, Brasília - DF. O teste de procedências foi instalado utilizando delineamento de blocos ao acaso com três repetições de 36 plantas, em espaçamento de $3 \times 2 \mathrm{~m}$. No plantio, utilizaram-se $200 \mathrm{~g}$ de superfosfato triplo, $50 \mathrm{~g}$ de FTE e $50 \mathrm{~g}$ de cloreto de potássio, em covas de $20 \times 20 \times 20 \mathrm{~cm}$. A partir do segundo ano, foram aplicados $50 \mathrm{~g}$ de ureia, $60 \mathrm{~g}$ de superfosfato triplo e $40 \mathrm{~g}$ de $\mathrm{KCl}$ em cobertura, três meses antes das colheitas.

O clima da região é tropical tipo Aw, quente e úmido, apresenta período seco bem definido com ocorrência de déficit hídrico, temperatura média anual de $25^{\circ} \mathrm{C}$, precipitação média anual de $2.354 \mathrm{~mm}$ e evapotranspiração média anual de $851 \mathrm{~mm}$, segundo as Normais Climatológicas (BRASIL, 1992). A maturação desuniforme dos frutos é uma característica dessa oleaginosa. Na região, a planta frutifica durante o período chuvoso, que vai de novembro a junho. $\mathrm{O}$ rendimento de grãos foi avaliado no $1^{\circ} \stackrel{\circ}{-} 2^{\circ}$ e $3^{\circ}$ ano pósplantio, com a realização de cinco colheitas (junho de 2009, dezembro de 2009, janeiro de 2010, junho de 2010 e dezembro de 2011). A colheita de junho de 2011 não foi realizada devido ao ataque severo de cigarrinha (Empoasca spp.), no período de florescimento e enchimento de grãos, de março a maio de 2011.

Os frutos em estágio final de maturação foram colhidos e secos à sombra por sete dias. Após o beneficiamento, a umidade das sementes foi mensurada em determinador Dole 500. Lotes de sementes com teor de umidade inferior a $9 \%$ foram pesados e anotados, resultando na produção de grãos (PG). O número de frutos maduros foi interpretado utilizando um índice de maturação (IM) estimado pela razão do número de frutos maduros e o total de frutos produzidos. Também foi computado o número de cachos (NC) e o número de frutos por cachos $(\mathrm{F} / \mathrm{C})$. Os seguintes caracteres vegetativos foram medidos em nível de indivíduo: número de ramos (NR), altura de plantas (ALT), projeção da copa no sentido do maior espaçamento (P1) e projeção da copa no sentido do menor espaçamento (P2).

A predição dos valores genéticos aditivos dos genitores foi realizada utilizando-se o programa SELEGEN - Seleção Genética Computadorizada. Para predição dos valores genéticos, foi considerado 
o modelo linear misto descrito a seguir: $Y=X r+Z g+$ $W p+e$, em Y que: $Y=$ vetor de dados; $r=$ é o vetor dos efeitos de repetição assumidos como fixos, somados à média geral; $g$ = é o vetor dos efeitos genotípicos de procedências, assumidos como aleatórios; $p=$ é o vetor de efeitos de parcelas assumidos como aleatórios; $e=$ vetor de erros aleatórios. As letras maiúsculas representam as matrizes de incidência para os respectivos efeitos (RESENDE, 2002).

Para seleção individual, foi considerada a média harmônica da performance relativa dos valores genéticos (MHPRVG) da produção de grãos, para selecionar simultaneamente produtividade e estabilidade. Esse método baseia-se em uma propriedade da média harmônica que favorece os genótipos de valor genético superior e que apresentam menor variação entre as colheitas (RESENDE, 2007).

\section{RESULTADOS E DISCUSSÃO}

A produção de grãos dessa oleaginosa é uma característica de herança complexa, cuja expressão depende da associação de diversas outras, tais como o número de ramos, de cachos por planta, de frutos por cacho, entre outras (SPINELLI et al., 2010). Observase que as características de produção apresentaram controle genético diferenciado (Tabela 1).

Entre os parâmetros genéticos de maior importância para a seleção de plantas, destacam-se os componentes de variância, em especial a variância genotípica (CRUZ et al., 2004). As características altura e número de ramos apresentaram controle genético predominante, indicando a possibilidade de obtenção de ganhos com a prática da seleção. LAVIOLA et al. (2012) observaram que características vegetativas apresentaram maiores estimativas de herdabilidade em sentido restrito.

A boa precisão experimental e acurácia em relação à análise dos dados foi indicada pelos baixos valores do coeficiente de determinação dos efeitos de parcelas $\left(\mathrm{C}_{\text {parc }}^{2}\right)$ e coeficiente de variação residual $\left(C V_{e}\right)$ para a maioria dos caracteres avaliados. No

Tabela 1 - Parâmetros genéticos estimados no $1^{\underline{0}}$ e $2^{\circ}$ anos pós-plantio dos principais componentes de produção e arquitetura da planta de Jatropha curcas, Porto Velho - RO.

\begin{tabular}{|c|c|c|c|c|c|c|c|}
\hline Parâmetros $\left(1^{\circ}\right.$ ano $)$ & $\begin{array}{c}\text { Número de } \\
\text { cachos }\end{array}$ & $\begin{array}{c}\text { Número de } \\
\text { frutos por } \\
\text { cacho }\end{array}$ & $\begin{array}{l}\text { Índice de } \\
\text { maturação }\end{array}$ & $\begin{array}{l}\text { Número de } \\
\text { ramos }\end{array}$ & $\begin{array}{l}\text { Altura de } \\
\text { plantas\# }\end{array}$ & $\begin{array}{l}\text { Projeção copa } \\
\text { maior } \\
\text { espaçamento\# }\end{array}$ & $\begin{array}{c}\text { Projeção copa } \\
\text { menor } \\
\text { espaçamento\# }\end{array}$ \\
\hline $\mathrm{V}_{\mathrm{g}}$ & 10,86 & 0,01 & 0,00 & 5,16 & 0,03 & 0,00 & 0,00 \\
\hline $\mathrm{V}_{\text {parc }}^{\mathrm{g}}$ & 2,58 & 0,05 & 0,00 & 0,38 & 0,00 & 0,00 & 0,00 \\
\hline $\mathrm{V}_{\mathrm{e}}$ & 69,95 & 0,61 & 0,04 & 8,80 & 0,03 & 0,05 & 0,04 \\
\hline$V_{f}$ & 83,41 & 0,68 & 0,04 & 14,35 & 0,06 & 0,06 & 0,05 \\
\hline $\mathrm{h}_{\mathrm{g}}^{2}$ & $\begin{array}{l}0,13 \\
(0,07)\end{array}$ & $\begin{array}{l}0,02 \\
(0,03)\end{array}$ & $\begin{array}{l}0,00 \\
(0,00)\end{array}$ & $\begin{array}{l}0,36 \\
(0,10)\end{array}$ & $\begin{array}{l}0,50 \\
(0,12)\end{array}$ & $\begin{array}{l}0,09 \\
(0,06)\end{array}$ & $\begin{array}{l}0,00 \\
(0,01)\end{array}$ \\
\hline $\mathrm{c}_{\text {parc }}^{2}$ & 0,03 & 0,07 & 0,00 & 0,02 & 0,02 & 0,00 & 0,14 \\
\hline $\mathrm{h}_{\mathrm{mp}}^{2}$ & 0,82 & 0,35 & 0,01 & 0,94 & 0,96 & 0,62 & 0,04 \\
\hline $\mathrm{A}_{\text {cproc }}$ & 0,90 & 0,59 & 0,12 & 0,97 & 0,98 & 0,79 & 0,20 \\
\hline $\mathrm{CV}_{\mathrm{e}}$ & 26,6 & 21,0 & 32,3 & 46,5 & 8,7 & 27,6 & 24,4 \\
\hline Média geral & 37,55 & 4,79 & 0,62 & 7,71 & 1,98 & 0,81 & 0,82 \\
\hline \multicolumn{8}{|l|}{ Parâmetros $\left(2^{\circ}\right.$ ano $)$} \\
\hline $\mathrm{V}_{\mathrm{g}}$ & 12,27 & 0,00 & 0,00 & 0,20 & 0,05 & 0,02 & 0,00 \\
\hline $\mathrm{V}_{\text {parc }}^{\mathrm{g}}$ & 3,06 & 0,01 & 0,00 & 5,76 & 0,00 & 0,00 & 0,00 \\
\hline $\mathrm{V}_{\mathrm{e}}$ & 71,95 & 0,26 & 0,01 & 24,59 & 0,04 & 0,08 & 0,12 \\
\hline $\mathrm{V}_{\mathrm{f}}$ & 87,28 & 0,28 & 0,01 & 30,56 & 0,09 & 0,11 & 0,14 \\
\hline $\mathrm{h}_{2 \mathrm{~g}}$ & $\begin{array}{l}0,14 \\
(0,06)\end{array}$ & $\begin{array}{l}0,00 \\
(0,00)\end{array}$ & $\begin{array}{l}0,00 \\
(0,00)\end{array}$ & $\begin{array}{l}0,00 \\
(0,01)\end{array}$ & $\begin{array}{l}0,50 \\
(0,12)\end{array}$ & $\begin{array}{l}0,23 \\
(0,09)\end{array}$ & $\begin{array}{l}0,06 \\
(0,05)\end{array}$ \\
\hline$c^{2}$ parc & 0,04 & 0,05 & 0,31 & 0,18 & 0,03 & 0,00 & 0,02 \\
\hline $\mathrm{h}_{\mathrm{mp}}^{2}$ & 0,82 & 0,02 & 0,02 & 0,07 & 0,95 & 0,73 & 0,71 \\
\hline $\mathrm{A}_{\text {cproc }}$ & 0,90 & 0,14 & 0,16 & 0,27 & 0,97 & 0,74 & 0,64 \\
\hline $\mathrm{CV}_{\mathrm{e}}$ & 40,6 & 17,7 & 13,5 & 29,8 & 7,1 & 9,7 & 12,2 \\
\hline Média geral & 25,22 & 3,45 & 0,74 & 24,70 & 2,81 & 2,93 & 2,83 \\
\hline
\end{tabular}

$\mathrm{V}_{\mathrm{g}}$ : variância genotípica, $\mathrm{V}_{\text {parc }}$ : variância ambiental entre parcelas, $\mathrm{V}_{\mathrm{e}}$ : variância residual, $\mathrm{V}_{\mathrm{f}}$ : variância fenotípica individual, $\mathrm{h}_{\mathrm{g}}^{2}$ : herdabilidade individual no sentido amplo, $\mathrm{c}^{2}$ parc: coeficiente de determinação dos efeitos da parcela, $\mathrm{h}^{2}$ mp: herdabilidade da média de progênies, $\mathrm{A}_{\text {cproc }}$ : acurácia de seleção, $\mathrm{CV}_{\mathrm{e}}$ : coeficiente de variação residual.

${ }^{\#}$ Unidade de medida utilizada metros 
presente trabalho, os valores de $\mathrm{C}^{2}$ parc encontraramse entre os limites de zero, para número de cachos, frutos por cacho, índice de maturação no $1^{\circ}$ e $2^{\circ}$ anos pós-plantio (Tabela 1).

Valores de $\mathrm{CV}_{\mathrm{e}}$ variaram de 7,1\% para a altura a $46,5 \%$ para número de ramos. $\mathrm{O}$ coeficiente de variação residual varia conforme o tipo de característica, delineamento experimental e espécie avaliada. Segundo RESENDE (2007), características provenientes de contagem como o número de ramos e o número de cachos apresentam maiores valores do coeficiente de variação. Para CRUZ et al. (2004), caracteres fenotípicos contínuos com valores inferiores a 20\% têm refletido boa precisão durante o desenvolvimento do experimento para diferentes espécies.

Em geral, herdabilidades individuais de baixa magnitude estão associadas às estimativas em nível de média de procedências de magnitudes moderada e alta $\left(\mathrm{h}^{2}{ }_{\mathrm{mp}} \geq 0,70\right)$ (RESENDE, 2007). Elevadas magnitudes de herdabilidades médias de procedências foram obtidas para as características vegetativas, indicando eficiência da seleção precoce entre e dentro de procedências (Tabela 1). LAVIOLA et al. (2012) encontraram valores de moderada e alta magnitude para a herdabilidade no sentido restrito para os caracteres vegetativos em plantas no $1^{\circ}$ e $2^{\circ}$ anos pós-plantio.

A acurácia seletiva refere-se à correlação entre os valores genéticos preditos e os valores genéticos verdadeiros dos indivíduos (RESENDE, 2007). Altos valores de herdabilidade em nível de médias de procedências proporcionaram altas acurácias seletivas, cujos valores variaram de 0.98 para altura a 0.12 para a maturação dos frutos (Tabela 1). Como a altura da planta é uma característica que influencia diretamente a colheita manual dos frutos, são desejáveis árvores de pinhãomanso de porte baixo. As estimativas de herdabilidade e de acurácia indicam que a seleção para altura pode ser realizada precocemente, nos primeiros anos de desenvolvimento.

A utilização de espaçamento de 2 metros entre plantas reduziu o crescimento da projeção de copa das plantas a partir do $2^{\circ}$ ano pós-plantio, tendo limitado a avaliação dessa característica (Tabelas 2 e 3). A avaliação de plantas em espaçamentos menos restritos mostra uma tendência contrária à observada, no caso de aumento da herdabilidade com o passar dos anos (GINWAL et al., 2005; RAO et al., 2009).

$\mathrm{O}$ índice de maturação dos frutos indica uma predominância do efeito ambiental no desenvolvimento dos frutos (Tabela 1). A desuniformidade de maturação dos frutos é uma
Tabela 2 - Parâmetros genéticos da produção de grãos de Jatropha curcas L., Porto Velho - RO.

\begin{tabular}{llll}
\hline Parâmetros & $1^{\underline{0} \text { ano }}$ & $2^{\underline{\underline{o}} \text { ano }}$ & $3^{\underline{0} \text { ano }}$ \\
\hline $\mathrm{V}_{\mathrm{g}}$ & 2320,63 & 8336,07 & 14889,50 \\
$\mathrm{~V}_{\text {parc }}$ & 47,84 & 3561,28 & 162,08 \\
$\mathrm{~V}_{\mathrm{e}}$ & 14808,30 & 69410,10 & 42756,90 \\
$\mathrm{~V}_{\mathrm{f}}$ & 17176,70 & 81307,40 & 57808,50 \\
& & & \\
$\mathrm{~h}_{\mathrm{g}}^{2}$ & 0,14 & 0,10 & 0,26 \\
& $(0,07)$ & $(0,06)$ & $(0,07)$ \\
$\mathrm{c}_{\text {parc }}$ & & & \\
$\mathrm{h}_{\text {mp }}^{2}$ & 0,00 & 0,04 & 0,00 \\
$\mathrm{~A}_{\text {cproc }}$ & 0,88 & 0,76 & 0,91 \\
$\mathrm{CV}_{\mathrm{e}}$ & 0,93 & 0,87 & 0,94 \\
Média geral & 0,29 & 0,35 & 0,39 \\
\hline
\end{tabular}

$\mathrm{V}_{\mathrm{g}}$ : variância genotípica, $\mathrm{V}_{\mathrm{parc}}$ : variância ambiental entre parcelas, $\mathrm{V}_{\mathrm{e}}$ : variância residual, $\mathrm{V}_{\mathrm{f}}$ : variância fenotípica individual, $\mathrm{h}_{\mathrm{g}}^{2}$ : herdabilidade individual no sentido amplo, $\mathrm{c}_{\text {parc }}^{2}$ : coeficiente de determinação dos efeitos da parcela, $\mathrm{h}^{2}{ }_{\mathrm{mp}}$ : herdabilidade da média de progênies, $A_{\text {cproc: }}$ acurácia de seleção, $C_{\mathrm{e}}$ : coeficiente de variação residual.

característica dessa oleaginosa, que floresce constantemente durante o período das chuvas, sendo a colheita realizada após o acúmulo de três a quatro floradas de maior vigor. A predominância do componente ambiental em relação ao componente genético indica pequenos ganhos com a seleção, e que práticas de manejo têm maior potencial para alterar esta característica (GHOSH et al., 2010; GOUVEIA et al., 2012).

A produção de grãos pode ser considerada a principal característica para seleção de plantas (SPINELLI et al., 2010; ROCHA et al., 2012). Avaliações agronômicas têm indicado a existência de variabilidade genética na expressão dessa característica, com produtividades variando de 0,2 a $2 \mathrm{~kg}$ planta $^{-1}$ de grãos, entre acessos (DIVAKARA et al., 2010). Projeções de produtividade baseadas em observações isoladas, ou de plantas com poucos meses de plantio, contribuíram para criar expectativas de produtividade não realizadas em campo (LAVIOLA et al., 2012). Além do efeito ambiental que influencia a produtividade final dos plantios, observou-se que, mesmo em um único ambiente, o plantio de materiais não selecionados é um importante fator de variação da produção de grãos.

Dos 432 genótipos avaliados no presente estudo, 60 deles $(13 \%)$ produziram menos de $300 \mathrm{~g}$ ano $^{-1}$ de frutos (Tabela 2), sem apresentar resposta à melhoria do ambiente. A procedência 3 
Tabela 3 - Estimativas dos efeitos genotípicos, progresso genético (g.planta ${ }^{-1}$ ) e acurácia com a prática da seleção entre procedências de Jatropha curcas, Porto Velho - RO.

\begin{tabular}{|c|c|c|c|c|c|}
\hline Ordem & Procededência. & g & $\mathrm{u}+\mathrm{g}$ & Ganho & Nova Média \\
\hline \multicolumn{6}{|c|}{ 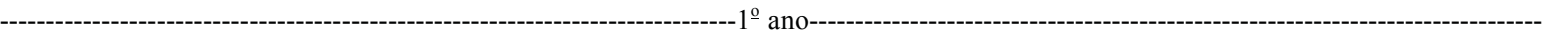 } \\
\hline 1 & 1 & 50,8 & 498,0 & 50,8 & 498,0 \\
\hline 2 & 4 & 23,0 & 470,3 & 36,9 & 484,2 \\
\hline 3 & 2 & $-25,0$ & 422,2 & 16,2 & 463,5 \\
\hline 4 & 3 & $-48,7$ & 398,5 & 0,0 & 447,3 \\
\hline 1 & 1 & 70,2 & 999,1 & 70,2 & 999,1 \\
\hline 2 & 4 & 51,8 & 980,7 & 61,0 & 989,9 \\
\hline 3 & 2 & $-16,7$ & 912,1 & 35,1 & 964,0 \\
\hline 4 & 3 & $-105,3$ & 823,6 & 0,0 & 928,9 \\
\hline 1 & 1 & 106,0 & 703,0 & 106,0 & 703,0 \\
\hline 2 & 2 & 26,0 & 623,0 & 66,0 & 663,1 \\
\hline 3 & 4 & $-1,0$ & 595,0 & 44,0 & 640,2 \\
\hline 4 & 3 & $-132,0$ & 465,0 & 0,0 & 596,6 \\
\hline
\end{tabular}

u: média geral, g: efeito genotípico.

Procedência 1e 2, de Rondônia; 3, de Minas Gerais e 4 de São Paulo.

apresentou as menores médias ao longo dos anos (Tabela 2). Melhorias nas condições do experimento foram obtidas pela calagem em área total e adubação química por cobertura, realizada antes das principais colheitas, conforme resultados das análises de solo (Dados não apresentados). Dois critérios foram considerados para a seleção: a seleção entre procedências e a seleção individual, considerando uma intensidade de seleção de 7\%, de acordo com RESENDE (2007). A procedência 1, oriunda de sementes do Estado de Rondônia, apresentou as maiores estimativas dos efeitos genotípicos preditos para a produção de grãos (Tabela 3). A seleção dessa procedência resulta na obtenção de ganhos de $11 \%, 7 \%$ e $18 \%$ no $1 \frac{0}{\text { o }} 2^{\circ}$ e e $3^{\circ}$ anos pós-plantio, respectivamente. A pequena alteração na média da população com a seleção de procedências concorda com os resultados encontrados por ROSADO et al. (2010), os quais observaram que a variabilidade genética dessa oleaginosa no país não se correlaciona com a localidade geográfica.

Embora a média dos efeitos genotípicos das outras procedências seja pequena, ou até mesmo negativa (Tabela 3), a predição dos valores genéticos individuais permite aumentar o ganho genético com a seleção dos melhores genótipos dentro de procedências. Em geral, a menor acurácia dos valores de herdabilidade observados para a unidade de seleção individual dentro de procedência não implica erro na classificação das matrizes, e sim nas estimativas do progresso genético (RESENDE, 2007). A seleção dos 30 melhores indivíduos resultou em um progresso genético de 29\% (Tabela 4). Foram selecionados 22 indivíduos da procedência 1 e 8 indivíduos da procedência 4 .

A seleção de plantas nas condições edafoclimáticas dos trópicos favorece a caracterização local de genótipos adaptados à temperatura e à umidade elevadas, maior irradiação solar e período seco bem definido. A verificação de que a uniformidade de maturação apresenta efeito ambiental predominante indica que práticas de manejo têm maior potencial para concentrar a colheita dessa oleaginosa. Trabalhos recentes indicam resultados promissores com a utilização de reguladores de crescimento para aumento da uniformidade de maturação e produção dessa oleaginosa (GHOSH et al. 2010; GOUVEIA et al., 2012). Também se destaca a boa adaptação dessa oleaginosa à região, que se caracteriza pela alta pluviosidade e déficit hídrico bem definido, que contribuem para uma colheita antecipada em relação a outras regiões do país, fora da época de incidência das principais pragas desse cultivo.

Os métodos de seleção e a variabilidade das populações influenciam no progresso genético a ser obtido com a seleção, quantificado a partir dos parâmetros genéticos avaliados neste trabalho. Embora acurado, o progresso genético estimado neste trabalho não parece ser suficiente para prover um incremento quantitativo na produtividade dessa oleaginosa. $\mathrm{O}$ desenvolvimento de novos materiais deve considerar estratégias de geração de variabilidade, tais como a recombinação entre indivíduos divergentes e a introdução de novos acessos. 
Tabela 4 - Ganhos genéticos preditos, considerando a produção de grãos anual (g planta ${ }^{-1}$ ), considerando a seleção dos 30 indivíduos de melhor desempenho, de acordo com a média harmônica da performance relativa dos valores genéticos (MHPRVG), Porto Velho $-\mathrm{RO}$.

\begin{tabular}{|c|c|c|c|c|c|c|c|c|c|}
\hline Ordem & Bloco & Proced. & Arvore & $1^{\underline{0}}$ ano & $2^{\underline{o}}$ ano & $3^{\circ}$ ano & MHPRVG & $\begin{array}{l}\text { Nova } \\
\text { Média }\end{array}$ & Ganho (\%) \\
\hline 1 & 3 & 1 & 14 & 570,0 & 1284,2 & 934,1 & 832,5 & 929,37 & 41 \\
\hline 2 & 1 & 1 & 10 & 568,9 & 1199,1 & 960,0 & 825,7 & 909,30 & 38 \\
\hline 3 & 3 & 1 & 4 & 568,4 & 1154,3 & 926,2 & 809,6 & 882,87 & 34 \\
\hline 4 & 1 & 1 & 16 & 558,7 & 1120,0 & 950,8 & 803,3 & 876,50 & 33 \\
\hline 5 & 3 & 1 & 5 & 555,8 & 1068,2 & 957,9 & 793,8 & 860,57 & 31 \\
\hline 6 & 2 & 4 & 16 & 558,0 & 1042,7 & 961,2 & 791,2 & 853,97 & 30 \\
\hline 7 & 3 & 4 & 10 & 567,6 & 1040,2 & 942,6 & 792,8 & 850,13 & 30 \\
\hline 10 & 1 & 1 & 11 & 544,7 & 1090,3 & 945,1 & 787,2 & 860,03 & 31 \\
\hline 15 & 3 & 1 & 2 & 557,0 & 1040,7 & 934,6 & 784,1 & 844,10 & 28 \\
\hline 20 & 3 & 1 & 7 & 546,6 & 1057,5 & 931,9 & 779,6 & 845,33 & 29 \\
\hline 30 & 1 & 1 & 12 & 533,1 & 1083,8 & 921,4 & 772,4 & 846,10 & 29 \\
\hline
\end{tabular}

\section{CONCLUSÃO}

As estimativas de variância genotípica indicam existir variabilidade genética com possibilidade de obter ganhos com a seleção de plantas. As características, número de frutos por cacho e a maturação de frutos não apresentaram controle genético predominante. $\mathrm{O}$ progresso genético estimado para a produção de grãos com a seleção de procedências pode ser considerado pequeno, indicando que a variabilidade genética não esteve associada à localização geográfica.

\section{AGRADECIMENTOS}

À Coordenação de aperfeiçoamento de Pessoal de Nível Superior (CAPES), pela bolsa de mestrado concedida. Ao Conselho Nacional de Desenvolvimento Científico e Tecnológico (CNPq) e a Fundação de Amparo a Pesquisa do Estado do Amazonas (FAPEAM), pelo apoio financeiro.

\section{REFERÊNCIAS}

BASHA, S.D. et al. A comparative study of biochemical traits and molecular markers for assessment of genetic relationships between Jatropha curcas L. germplasm from different countries. Plant Science, v.176, n.6, p.812-823, 2009. Disponível em: <http://www. sciencedirect.com/science/article/pii/SO168945209000934>. Acesso em: 13 jan. 2013. doi:10.1016/j.plantsci.2009.03.008.

BHERING, L.L. et al. Differential response of Jatropha genotypes to different selection methods indicates that combined selection is more suited than other methods for rapid improvement of the species. Industrial Crops and Products, v.41, p.260-265, 2013. Disponível em: <http://www.scincedirect.com/science/article/pii/ S0926669012002166>. Acesso em 13 jan. 2013. doi:10.1016/j. indcrop.2012.04.026.

BRASIL. Normais climatológicas: 1961-1990. METEOROLOGIA., M.D.A.E.D.R.A.D.N.D. Brasil. Brasília, DF, 1992. 84p.
CRUZ, C.D. et al. Modelos biométricos aplicados ao melhoramento genético. Viçosa: UFV, 2004. V.1, 480p.

CRUZ, C.D.; CARNEIRO, P.C.S. Modelos biométricos aplicados ao melhoramento genético. Viçosa: UFV, 2003. 585p.

DIVAKARA, B.N. et al. Biology and genetic improvement of Jatropha curcas L.: a review. Applied Energy, v.87, n.3, p.732742, 2010. Disponível em: <http//www.sciencedirect.com/science/ article/pii/S030626190900302X > Acesso em: 13 jan. 2013. doi: 10.1016/j. apenergy.2009.07.013.

GHOSH, A. et al. Paclobutrazol arrests vegetative growth and unveils unexpressed yield potential of Jatropha curcas. Journal of Plant Growth Regulation, v.29, n.3, p.307-315, 2010. Disponível em: <http://jatropha.pro/PDF\%20bestanden/cultar. pdf $>$. Acesso em: 13 jan. 2013. doi. 10.1007/s00344-010-9137-0.

GINWAL, H.S. et al. Seed source variation in morphology, germination and seedling growth of Jatropha curcas Linn. in central India. Silvae Genetica, v.54, n.2, p.76-80, 2005. Disponível em: <http://www.allgemeineforstundjagdzeitung.de>. Acesso em: 13 jan. 2013.

GOUVEIA, E.J. et al. Aumento da produção de grãos de pinhãomanso pela aplicação de benziladenina. Pesquisa Agropecuaria Brasileira, v.47, p.1541-1545, 2012. Disponível em: <http://www. alice.cnptia.embrapa.br>. Acesso em: 13 jan. 2013. doi: 10.1590/ S0100-204X2012001000018.

LAVIOLA, B.G. et al. Morpho-agronomic characterization of the germplasm bank of Jatropha young stage. Bioscience Journal, v.27, n.3, p.371-379, 2011. Disponível em: <http://www.seer.ufu. br>. Acesso em: 13 jan. 2013.

LAVIOLA, B.G. et al. Estimates of genetic parameters for physic nut traits based in the germplasm two years evaluation. Ciencia Rural, v.42, n.3, p.429-435, 2012. Disponível em: <http://www.scielo.br/ scielo.phd?pid. $=$ S0103-84782012000300008\&script $=$ sci_arttext $>$ Acesso em: 13 jan. 2013. doi: 10.1590/S0103-84782012000300008

LAVIOLA, B.G. et al. The importance of Jatropha for Brazil. In: CARELS, N. et al. Jatropha, challenges for a new energy crop. Verlag: Springer, 2012. Cap.5, p.71-94. 
LIN, C.S.; BINNS, M.R.A. Superiority measure of cultivar performance for cultivar $\mathrm{x}$ location data. Canadian Journal of Plant Science, v.68, p.193-198, 1988. Disponível em: <http:// pubs.aic.ca?doi/abs/10.4141/cjps88-018>. Acesso em: 13 jan. 2013. doi: $10.4141 /$ cjps88-018.

RAO, M.R.G. et al. Genetic diversity in Jatropha (Jatropha curcas L.). Research on Crops, v.10, n.2, p.420-425, 2009. Disponivel em : <http://www.cropresearch.org>. Acesso em 13 jan. 2013.

RESENDE, M.D.V. Genética biométrica e estatística no melhoramento de plantas perenes. Brasília: Embrapa Informação Tecnológica, 2002. 975p.

RESENDE, M.D.V. Matemática e estatística na análise de experimentos e no melhoramento genético. Colombo: Embrapa Florestas, 2007. 359p

ROCHA, R.B. et al. Selection efficiency for increasing physic nut oil content. Pesquisa Agropecuaria Brasileira, v.47, n.1, p.44-50, 2012. Disponível em: <http://www.scielo.br/scielo.
php?script=sci_arttext\&pid=S0100-204X2012000100007\&Ing= pt\&nrm=isso $>$. Acesso em: 13 jan. 2013. doi: 10.1590/S0100$204 X 2012000100007$.

ROCHA, R.B. et al. Avaliação genética de procedências de bandarra (Schizolobium amazonicum) utilizando REML/BLUP (Máxima verossimilhança restrita/Melhor predição linear não viciada). Scientia Forestalis, v.37, p.351-358, 2009. Disponível em: $<$ http://www.ipef.br $>$. Acesso em: 13 jan. 2013.

ROSADO, T.B. et al. Molecular markers reveal limited genetic diversity in a large germplasm collection of the biofuel crop Jatropha curcas L. in Brazil. Crop Science, v.50, n.6, p.23722382, 2010. Disponível em: <http://www.alice.cnptia.embrapa. br/handle/doc/864160>. Acesso em: 13 jan. 2013. doi: 10.2135/ cropsci2010.02.011.

SPINELLI, V.M. et al. Primary and secondary yield components of the oil in physic nut (Jatropha curcas L.). Ciencia Rural, v.40, n.8, p.1752-1758, 2010. Disponível em: $<$ http://scielo.br/pdf/cr/ v40n8/a687cr3156.pdf $>$. Acesso em: 13 jan. 2013. doi:10.1590/ S0103-84782010005000129. 